10,11

\title{
Влияние дейтерирования на фазовые переходы в диоксотетрафториде ванадия
}

\author{
(C) Е.В. Богданов ${ }^{1,2}$, Е.И. Погорельцев ${ }^{1,3}$, М.В. Горев ${ }^{1,3}$, М.С. Молокеев ${ }^{1,3}$, И.Н. Флеров ${ }^{1,3}$ \\ ${ }^{1}$ Институт фозики им. Л.В. Киренского СО РАН - обособленное подразделение ФИЦ КНЦ СО РАН, \\ Красноярск, Россия \\ ${ }^{2}$ Институт инженерных систем и энергетики, Красноярский аграрный университет, \\ Красноярск, Россия \\ ${ }^{3}$ Институт инженерной физики и радиоэлектроники, Сибирский фредеральный университет, \\ Красноярск, Россия \\ E-mail: evbogdanov@iph.krasn.ru
}

(Поступила в Редакцию 13 августа 2018 г.)

Выращены кристаллы $\left(\mathrm{ND}_{4}\right)_{3} \mathrm{VO}_{2} \mathrm{~F}_{4}$ с высокой степенью дейтерирования $\sim 87 \%$ и установлено значительное увеличение объема элементарной ячейки $(\sim 1.5 \%)$. Уменьшение химического давления привело к изменению последовательности фазовых переходов за счет выклинивания одной из ромбических фаз, наблюдавшейся в $\left(\mathrm{NH}_{4}\right)_{3} \mathrm{VO}_{2} \mathrm{~F}_{4}$. Выполнены теплофизические исследования и определены энтропии, деформации и барические коэффициенты, связанные со структурными превращениями. Построена фазовая $T$-p-диаграмма и определены барические и температурные границы устойчивости кристаллических фаз. Диэлектрические исследования свидетельствуют о несегнетоэлектрической природе фазовых переходов в $\left(\mathrm{ND}_{4}\right)_{3} \mathrm{VO}_{2} \mathrm{~F}_{4}$. Выполнен сравнительный анализ экспериментальных и модельных энтропий. На основе уменьшения энтропии в результате дейтерирования предложена гипотеза о значительном, но не предельном, соответствующем разупорядочению, ангармонизме колебаний аммонийных тетраэдров.

Исследования выполнены при финансовой поддержке Российского фонда фундаментальных исследований, Правительства Красноярского края, Красноярского краевого фонда науки в рамках научного проекта: „Влияние дейтерирования на ориентационное упорядочение и фазовые переходы в аммонийных фторкислородных ванадатах“ № 18-42-243003.

DOI: $10.21883 /$ FTT.2019.02.47134.230

\section{1. Введение}

Для создания новых полярных материалов исследователи часто используют полярные базовые блоки, так как их наличие в структуре может привести к появлению макроскопического дипольного момента. Использование асимметричных блоков, таких как полиэдры переходных металлов или одиночные пары катионов, оказались успешными при синтезе новых полярных материалов с нелинейно-оптической активностью [1-5].

Кристаллическая структура комплексных оксифторидов состоит из полярных анионов $\left[\mathrm{Me}_{x} \mathrm{~F}_{6-x}\right] \quad[6]$. Фторкислородные октаэдры могут формировать различные конфигурации: линейные $\left(\mathrm{CuNbOF}_{5}\left(\mathrm{H}_{2} \mathrm{O}\right)(\text { pyz })_{3}\right.$ [7]) и зигзагообразные $\left(\mathrm{CuWO}_{2} \mathrm{~F}_{4}\left(\mathrm{H}_{2} \mathrm{O}\right)_{2}(p y)_{2}[8]\right)$ цепочки или иметь спиральный мотив $\left(\mathrm{AMoO}_{2} \mathrm{~F}_{4}\left(\mathrm{H}_{2} \mathrm{O}\right)_{2}(p y z)[9]\right)$. Причем спиральные цепи, как правило, упорядочены, зигзагообразные цепи разупорядочены, а линейные цепочки октаэдров $\left[\mathrm{Me}_{x} \mathrm{~F}_{6-x}\right]$ обладают либо ориентационным порядком, либо беспорядком. Разупорядочение фторкислородных октаэдров вызвано близкими размерами и электроотрицательностью кислородных и фторных анионов. Методология конструирования упорядоченных оксифторидных материалов заключается в создании изотропных сред вокруг фторкислородных октаэдров, которые приводили бы к упорядочению атомов кислорода и/или фтора. Для этого обычно используют оптимизацию пространства межоктаэдрических пустот [10], сопоставимость поляризуемостей [11] и/или внедрение разных катионов [12].

Оксифториды ванадия (VOF), благодаря способности атома ванадия менять валентные состояния, могут формировать различные искаженные фторкислородные октаэдры [13]. Наличие двух разных связующих мотивов вокруг центрального атома ванадия (связь $\mathrm{V}-\mathrm{O}$ и связь $\mathrm{V}-\mathrm{F}$ ) создает высокосимметричные локальные координационные сферы, которые могут привести к нелинейному оптическому поведению, такому как генерация второй оптической гармоники [14].

Однако, несмотря на многообразие оксифторидов ванадия (VOF), в большинстве из них фторкислородный анион является разупорядоченным, полностью упорядоченное состояние наблюдалось только в кристаллах $\mathrm{K}_{2} \mathrm{VO}_{2} \mathrm{~F}_{3}$ [15], структура которых состоит цепочек соединенных углами октаэдров VOF. Сегнетоэлектрическое и протонпроводящее состояния обнаружены в оксифториде ванадия $\left(\mathrm{NH}_{4}, \mathrm{~K}\right)_{3} \mathrm{VO}_{2} \mathrm{~F}_{4}$ [16]. Соединение относится к семейству эльпасолитов и при комнатной температуре имеет ромбическую симметрию (пр. гр. Immm).

Симметрия кристалла $\left(\mathrm{NH}_{4}\right)_{3} \mathrm{VO}_{2} \mathrm{~F}_{4}$ при комнатной температуре первоначально была определена как ромбическая с двумя равновероятными пространственны- 
ми группами Immm и I222, но предпочтение было отдано авторами первому варианту [17]. Фазовые переходы в этом оксифториде были подробно изучены в $[18,19]$. В соответствии с данными оптических исследований $\left(\mathrm{NH}_{4}\right)_{3} \mathrm{VO}_{2} \mathrm{~F}_{4}$ претерпевает четыре последовательных фазовых перехода со следующим изменением симметрии $F m \overline{3} m \leftrightarrow \operatorname{Immm}(I 222) \leftrightarrow$ ромбическая $\leftrightarrow P 112 / m \leftrightarrow P \overline{1}$ [18]. Калориметрические измерения подтвердили наличие фазовых превращений первого рода при температурах: $T_{1}=438 \mathrm{~K}, T_{2}=244 \mathrm{~K}, T_{3}=210 \mathrm{~K}$ и $T_{4}=205 \mathrm{~K}$. Анализ энтропий превращений позволил скорректировать модель ориентационного разупорядочения VOF-анионов в кубической фазе [17]. Учет разупорядочения атомов ванадия в процессе уточнения кристаллической структуры $\left(\mathrm{NH}_{4}\right)_{3} \mathrm{VO}_{2} \mathrm{~F}_{4}$ в монокристалльных рентгеновских исследованиях позволил с большой долей вероятности постулировать пр. гр. I222 для ромбической фазы, существующей в интервале $T_{1}>T>T_{2}$ [20]. Аммонийные тетраэдры и фторкислородные октаэдры предполагались разупорядоченными и частично упорядоченными соответственно в исходной кубической $F m \overline{3} m-$ и первой искаженной I222-фазах. Появление при $T<T_{2}$ двойников различной ориентации препятствовало уточнению структуры второй ромбической, моноклинной и триклинной фаз $[17,20]$.

К вопросу о роли катион/анионных полиэдров в механизме структурных искажений при фазовых переходах в комплексных фторидах и оксифторидах исследователи обращались неоднократно [21-23]. Существенная роль аммонийного катиона в механизмах разупорядочения/упорядочения фторкислородных структур с изолированными октаэдрами $\left(\mathrm{NH}_{4}\right)_{2} M e^{6+} \mathrm{O}_{2} \mathrm{~F}_{4}\left(M e^{6+}: \mathrm{W}\right.$, Мо) установлена при исследованиях соединений, синтезированных путем как замещения аммонийной группы одновалентным катионом [24], так и ее дейтерирования $[25,26]$.

В настоящей работе впервые выполнены исследования влияния дейтерирования аммонийного катиона на процессы разупорядочения/упорядочения, которые наблюдаются в оксифторидах со связанными анионными полиэдрами, на примере кристалла диоксотетрафториде ванадия $(\mathrm{V})-\left(\mathrm{ND}_{4}\right)_{3} \mathrm{VO}_{2} \mathrm{~F}_{4}$.

\section{2. Экспериментальные методы и результаты исследований}

Кристаллизация исходного соединения $\left(\mathrm{NH}_{4}\right)_{3} \mathrm{VO}_{2} \mathrm{~F}_{4}$ осуществлялась фторированием аммония ванадиевокислого с добавлением аммиака при температуре $T \approx 150-200$ C $[20]$

$$
\mathrm{NH}_{4} \mathrm{VO}_{3}+2 \cdot \mathrm{NH}_{4} \mathrm{OH}+4 \cdot \mathrm{HF}=\left(\mathrm{NH}_{4}\right)_{3} \mathrm{VO}_{2} \mathrm{~F}_{4}+3 \cdot \mathrm{H}_{2} \mathrm{O} .
$$

Полученные кристаллы $\left(\mathrm{NH}_{4}\right)_{3} \mathrm{VO}_{2} \mathrm{~F}_{4}$ растворялись в тяжелой воде $(99.9 \%$ D). Раствор выдерживался в эксикаторе с пентаоксидом фосфора $\mathrm{P}_{2} \mathrm{O}_{5}$ до полного
Таблица 1. Основные параметры уточнения структуры кристалла $\left(\mathrm{ND}_{4}\right)_{3} \mathrm{VO}_{2} \mathrm{~F}_{4}$

\begin{tabular}{c|c}
\hline Соединение & $\left(\mathrm{ND}_{4}\right)_{3} \mathrm{VO}_{2} \mathrm{~F}_{4}$ \\
\hline Sp.Gr. & Immm \\
$a, \AA$ & $9.1334(6)$ \\
$b, \AA$ & $18.833(1)$ \\
$c, \AA$ & $6.2701(5)$ \\
$V, \AA^{3}$ & $1078.5(1)$ \\
$Z$ & 6 \\
$2 \theta$-interval, & $5-90$ \\
$R_{\text {wp }}, \%$ & 16.10 \\
$R_{p}, \%$ & 12.44 \\
$R_{\text {exp }}, \%$ & 14.45 \\
$\chi^{2}$ & 1.11 \\
$R_{B}, \%$ & 3.83
\end{tabular}

поглощения воды и начала кристаллизации соединения. Для увеличения степени замещения $D \rightarrow H$ производилась многократная перекристаллизация. Степень дейтерирования контролировалась в каждом цикле путем сравнения интегральных линий поглощения ЯМР ${ }^{1} \mathrm{H}$ протонированного и дейтерированного соединений. В результате были получены небольшие $\left(\sim 1 \mathrm{~mm}^{3}\right)$ кристаллы $\left(\mathrm{ND}_{4}\right)_{3} \mathrm{VO}_{2} \mathrm{~F}_{4}$, имеющие желтую окраску, степень дейтерирования которых составляла $\sim 87 \%$.

Данные о структуре $\left(\mathrm{ND}_{4}\right)_{3} \mathrm{VO}_{2} \mathrm{~F}_{4}$ при комнатной температуре получены при помощи порошкового дифрактометра Bruker D8 ADVANCE (излучение $\left.\mathrm{Cu}-K_{\alpha}\right)$ и линейного детектора VANTEC $\left(2 \theta \approx 0.016^{\circ}, t \approx 0.5 \mathrm{~s} / 2 \theta\right)$.

Уточнение модели структуры методом Ритвельда (рис. 1) проводилось с использованием программы TOPAS 4.2 [27]. Основные пики были проиндицированы в ромбической ячейке. Отдать предпочтение какой-либо из пространственных групп, Immm или I222, не представлялось возможным. В табл. 1 приведены параметры ячейки для пр.гр. Immm, которые оказались близкими к параметрам, найденным для исходного кристалла $\left(\mathrm{NH}_{4}\right)_{3} \mathrm{VO}_{2} \mathrm{~F}_{4}$ [17,20]. Окончательное уточнение структуры привело к низким значениям $R$-факторов (табл. 1 ).

Для определения влияния дейтерирования на температуры и энергетические параметры фазовых переходов были выполнены исследования теплоемкости кристаллов $\left(\mathrm{ND}_{4}\right)_{3} \mathrm{VO}_{2} \mathrm{~F}_{4}$. В низкотемпературной области $T \approx 95-310 \mathrm{~K}$ измерения выполнены прецизионными методами адиабатической калориметрии. Образец массой $m \approx 1.2 \mathrm{~g}$ герметично упаковывался в индиевую капсулу в инертной атмосфере гелия. Калориметрические измерения проводились в режимах непрерывных $(d T / d t \approx 0.15 \mathrm{~K} / \mathrm{min})$ и дискретных $(\Delta T \approx 2.5-3.0 \mathrm{~K})$ нагревов. Теплоемкость фурнитуры, состоящей из нагревателя и индиевой капсулы, измерялась в отдельном эксперименте.

Исследования при высоких температурах $T \approx$ $\approx 315-460 \mathrm{~K}$ проводились методом дифференциальной 


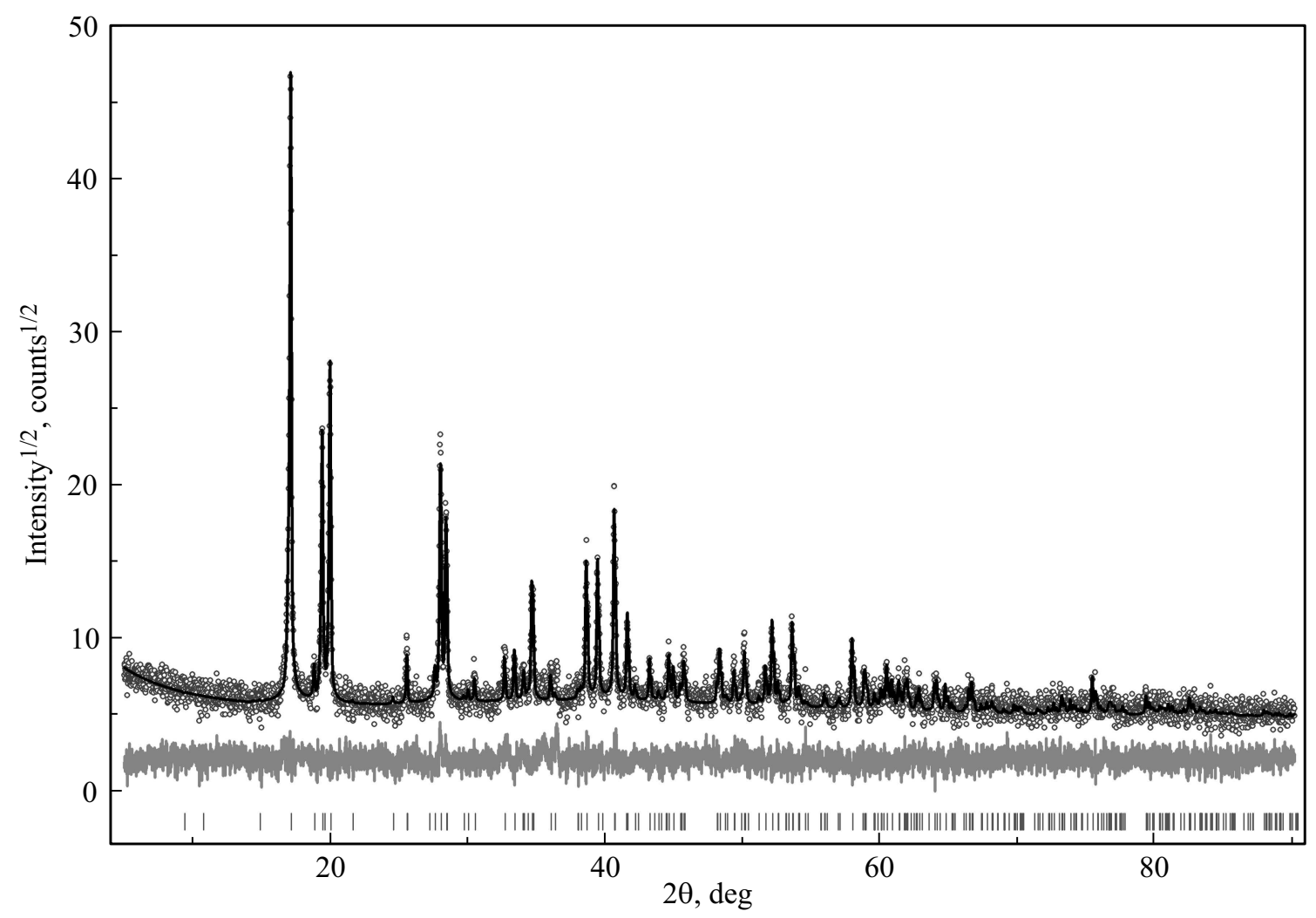

Рис. 1. Результаты уточнения модели структуры $\left(\mathrm{ND}_{4}\right)_{3} \mathrm{VO}_{2} \mathrm{~F}_{4}$ методом Ритвельда.

сканирующей калориметрии (NETZSCH $204 \quad F 1)$. Образец массой $m \approx 0.025 \mathrm{~g}$ помещался в алюминиевый контейнер. Измерения проводились в потоке гелия $(20 \mathrm{ml} / \mathrm{min})$ со скоростью нагрева $d T / d t \approx 5 \mathrm{~K} / \mathrm{min}$. Для определения абсолютных значений теплоемкости образца использовался кварцевый эталон.

На температурной зависимости теплоемкости кристалла $\left(\mathrm{ND}_{4}\right)_{3} \mathrm{VO}_{2} \mathrm{~F}_{4}$ обнаружены три аномалии $C_{p}$ (рис. 2,) при температурах, близких к температурам

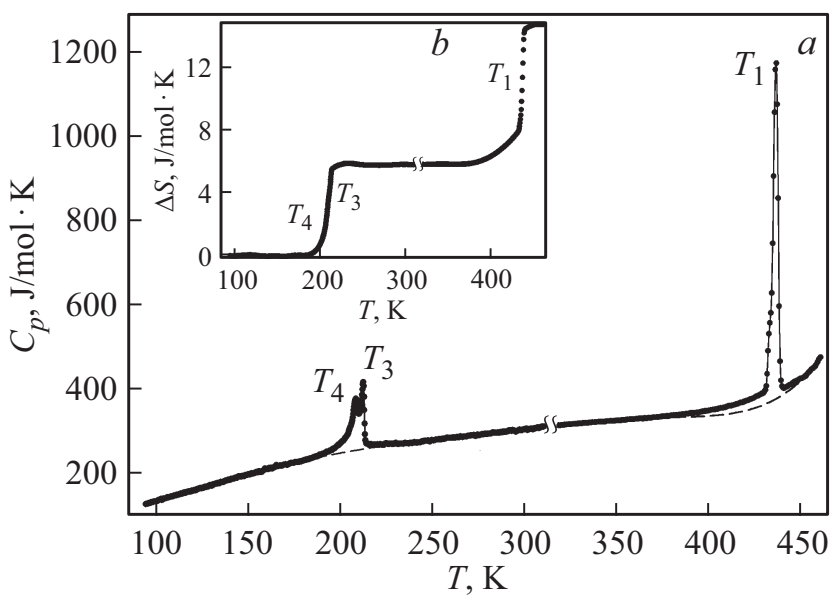

Рис. 2. Температурные зависимости теплоемкости $(a)$ и энтропии фазовых переходов $(b)$ в кристалле $\left(\mathrm{ND}_{4}\right)_{3} \mathrm{VO}_{2} \mathrm{~F}_{4}$ в широком интервале температур. фазовых переходов $T_{1}, T_{3}$ и $T_{4}$, наблюдавшимся в кристалле $\left(\mathrm{NH}_{4}\right)_{3} \mathrm{VO}_{2} \mathrm{~F}_{4}$ (табл. 2). Таким образом, аномалия, наблюдавшаяся в протонированном соединении в районе $T \approx 244 \mathrm{~K}$ [19], в дейтерированном кристалле не обнаружена, то есть в нем, судя по всему, отсутствует фазовый переход Immm $(I 222) \leftrightarrow$ ромбическая. Поэтому для удобства сравнения характеристик переходов низкотемпературным пикам $C_{p}(T)$ кристалла $\left(\mathrm{ND}_{4}\right)_{3} \mathrm{VO}_{2} \mathrm{~F}_{4}$ присвоены индексы $T_{3}$ и $T_{4}$ (табл. 2).

Аномальный рост $C_{p}(T)$, наблюдающийся при $T>440 \mathrm{~K}$, связан с началом процесса разложения кристалла, который интенсивно развивается при температуре $T^{\prime} \approx 480 \mathrm{~K}$ и сопровождается уменьшением массы образца. Для получения надежных сведений о термодинамических параметрах фазового перехода при $T_{1}$ измерения теплоемкости проводились на нескольких образцах.

Для определения интегральных характеристик фазовых переходов выполнено разделение полной теплоемкости $C_{p}$ на регулярную решеточную $C_{l}$ и аномальную $\Delta C_{p}=C_{p}-C_{l}$ составляющие. Экспериментальные данные $C_{p}(T)$ вдали от аномальных температурных областей интерполировались полиномиальной зависимостью с экстраполяцией на области фазовых переходов (рис. 2,a).

Интегрирование избыточной теплоемкости $\Delta C_{p}(T)$ позволило получить информацию об изменениях энтальпии, связанных с высокотемпературным фазовым 
Таблица 2. Некоторые термодинамические параметры фазовых переходов в оксифторидах $\left(\mathrm{ND}_{4}\right)_{3} \mathrm{VO}_{2} \mathrm{~F}_{4}$ и $\left(\mathrm{NH}_{4}\right)_{3} \mathrm{VO}_{2} \mathrm{~F}_{4} \quad[19] \quad\left(T_{C_{i}}-\right.$ температура Кюри, $N_{i}-$ степень близости фазового перехода к трикритической точке)

\begin{tabular}{l|c|c}
\hline \multicolumn{1}{c|}{ Параметр } & $\left(\mathrm{ND}_{4}\right)_{3} \mathrm{VO}_{2} \mathrm{~F}_{4}$ & $\left(\mathrm{NH}_{4}\right)_{3} \mathrm{VO}_{2} \mathrm{~F}_{4}[19]$ \\
\hline$T_{1}, \mathrm{~K}$ & $436.6 \pm 0.5$ & $438 \pm 1$ \\
$\Delta S_{1}, \mathrm{~J} / \mathrm{mol} \cdot \mathrm{K}$ & $8.7 \pm 0.9$ & $9.7 \pm 1.0$ \\
$\left(d T_{1} / d p\right)_{\text {расч }}, \mathrm{K} \cdot \mathrm{GPa}^{-1}$ & 130 & - \\
$T_{2}, \mathrm{~K}$ & - & $244.0 \pm 0.2$ \\
$\Delta S_{2}, \mathrm{~J} / \mathrm{mol} \cdot \mathrm{K}$ & - & $0.18 \pm 0.04$ \\
$T_{3}, \mathrm{~K}$ & $212.10 \pm 0.05$ & $210.2 \pm 0.2$ \\
$\left(A_{T}^{2} / B\right)_{T 3}, \mathrm{~J} / \mathrm{mol} \cdot \mathrm{K}^{2}$ & -0.8 & -0.4 \\
$\left(A_{T}^{3} / C\right)_{T 3}, \mathrm{~J} / \mathrm{mol} \cdot \mathrm{K}^{3}$ & 5.8 & 1.1 \\
$T_{3}-T_{C 3}, \mathrm{~K}$ & 2.2 & 1.4 \\
$N_{3}$ & -0.12 & -0.1 \\
$\Delta S_{3}, \mathrm{~J} / \mathrm{mol} \cdot \mathrm{K}$ & $4.8 \pm 0.6$ & $6.2 \pm 0.7$ \\
$\left(d T_{3} / d p\right)_{\text {эксп }}, \mathrm{K} \cdot \mathrm{GPa}^{-1}$ & $-43 \pm 5$ & $-27 \pm 3$ \\
$\left(d T_{3} / d p\right)_{\text {расч }}, \mathrm{K} \cdot \mathrm{GPa}^{-1}$ & $-40 \pm 10$ & - \\
$T_{4}, \mathrm{~K}$ & $208.1 \pm 0.1$ & $205.1 \pm 0.2$ \\
$\left(A_{T}^{2} / B\right)_{T 4}, \mathrm{~J} / \mathrm{mol} \cdot \mathrm{K}^{2}$ & -2.8 & -1.3 \\
$\left(A_{T}^{3} / C\right)_{T 4}, \mathrm{~J} / \mathrm{mol}^{2} \cdot \mathrm{K}^{3}$ & 2.8 & 2.2 \\
$T_{4}-T_{C 4}, \mathrm{~K}$ & 0.1 & 0.4 \\
$N_{4}$ & -0.02 & -0.05 \\
$\Delta S_{4}, \mathrm{~J} / \mathrm{mol} \cdot \mathrm{K}$ & $1.00 \pm 0.12$ & $0.80 \pm 0.12$ \\
$\left(d T_{4} / d p\right)_{\text {эксп }}, \mathrm{K} \cdot \mathrm{GPa}^{-1}$ & $-77 \pm 5$ & $-25 \pm 3$ \\
$\left(d T_{4} / d p\right)_{\text {расч }}, \mathrm{K} \cdot \mathrm{GPa}^{-1}$ & $-70 \pm 20$ & - \\
& &
\end{tabular}

переходом $\Delta H_{1}=3700 \pm 350 \mathrm{~J} / \mathrm{mol}$ и последовательностью низкотемпературных превращений $\Delta H_{3}+\Delta H_{4}=$ $=1200 \pm 90 \mathrm{~J} / \mathrm{mol}$. Определение в последнем случае только суммарной величины $\Sigma \Delta H$ обусловлено затруднениями выделения индивидуальных энтальпийных вкладовиз-за очень близких значений $T_{3}$ и $T_{4}$.

Температурное поведение линейного теплового расширения исследовано в интервале температур $T \approx$ $\approx 120-485 \mathrm{~K}$ на дилатометре NETZSCH DIL402C со скоростью нагревов $d T / d t=3 \mathrm{~K} / \mathrm{min}$. Измерения проводились в инертной атмосфере гелия (скорость потока $50 \mathrm{ml} / \mathrm{min}$ ). Тепловое расширение измерительной системы было определено в отдельном эксперименте на эталонном образце кварца. Образцы для дилатометрических экспериментов были приготовлены в виде таблеток $(d=8 \mathrm{~mm}, h=2-3 \mathrm{~mm})$ путем прессования $(\Delta p \approx 2 \mathrm{GPa})$ без последующей термообработки ввиду наличия в исследуемом соединении аммонийного катиона.

На температурных зависимостях объемной деформации $\Delta V / V_{0}=3 \cdot\left(\Delta L / L_{0}\right)$ (рис. $\left.3, a\right)$ и коэффициента объемного теплового расширения $\beta=3 \cdot \alpha$ (рис. $3, b$ и $3, c)$ наблюдается аномальное поведение в области температур фазовых переходов, установленных в калориметрических экспериментах. Обнаружено, что характер аномалий зависит от термической предыстории образца и наличия/отсутствия в нем механических напряжений. При первом нагреве образца в исходном напряжен-

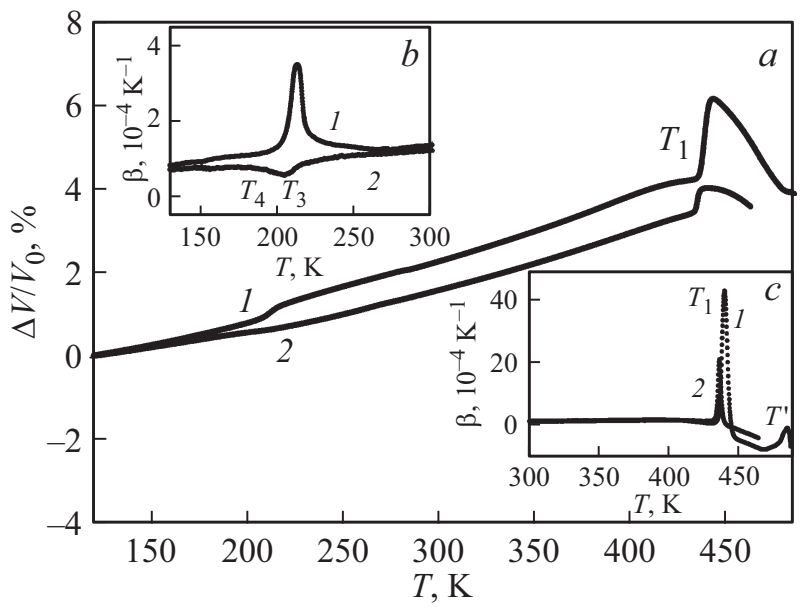

Рис. 3. Температурные зависимости: спонтанной деформации кристалла $\left(\mathrm{ND}_{4}\right)_{3} \mathrm{VO}_{2} \mathrm{~F}_{4}$ в исходном напряженном состоянии $(1)$ и после отжига при $T>T_{1}(2)(a)$ и коэффициента объемного расширения в низкотемпературной $(b)$ и высокотемпературной $(c)$ областях.

ном состоянии, возникающем при прессовании таблеток, наблюдалось значительное скачкообразное увеличение деформации $\delta\left(\Delta V / V_{0}\right) \approx 2 \%$ при температуре $T_{1}$ и $\delta\left(\Delta V / V_{0}\right) \approx 0.5 \%$ в районе $T_{3}$ и $T_{4}$ (кривая $(1)$ на рис. $3, a)$. При нагреве до температур $T_{1}<T<450 \mathrm{~K}$, по-видимому, происходит отжиг образца, о чем свидетельствуют результаты повторных измерений. Наблюдалось значительное уменьшение скачка $\delta\left(\Delta V / V_{0}\right)$ при $T_{1}$ и изменение знака на отрицательный для аномалий при $T_{3}$ и $T_{4}:\left(\delta V / V_{0}\right)_{3} \approx-0.19 \%$ и $\left(\delta V / V_{0}\right)_{4} \approx-0.07 \%$ (кривая (2) на рис. 3,a).

При нагревании кристалла $\left(\mathrm{ND}_{4}\right)_{3} \mathrm{VO}_{2} \mathrm{~F}_{4}$ до $T>450 \mathrm{~K}$ наблюдается аномальное поведение $\beta(T)$ с максимумом при температуре $T^{\prime} \approx 480 \mathrm{~K}$ (рис. $\left.3, b\right)$, при которой происходит разложение кристаллов, наблюдавшееся также в калориметрических измерениях.

Восприимчивость температур фазовых переходов к давлению изучалась методом дифференциального термического анализа (ДТА). Гидростатическое давление $(p \leq 0.5 \mathrm{GPa})$ создавалось в сосуде поршень-цилиндр с мультипликатором с использованием силиконового масла в измерительной камере. Масса образца составляла $m \approx 0.025 \mathrm{~g}$. В качестве ДТА-термоэлемента использовалась медь-германиевая термопара, а эталоном служил образец кварца.

Специфические особенности конструкции ДТА-установки не позволили выполнить измерения в области высокотемпературного фазового перехода. В процессе низкотемпературных исследований при атмосферном давлении зафиксированы две аномалии ДТА-сигнала при температурах $T_{3}=212 \pm 1 \mathrm{~K}$ и $T_{4}=209 \pm 1 \mathrm{~K}$ (рис. 4), удовлетворительно согласующихся с данными калориметрических и дилатометрических измерений (табл. 2). Увеличение давления приводит к понижению темпера- 


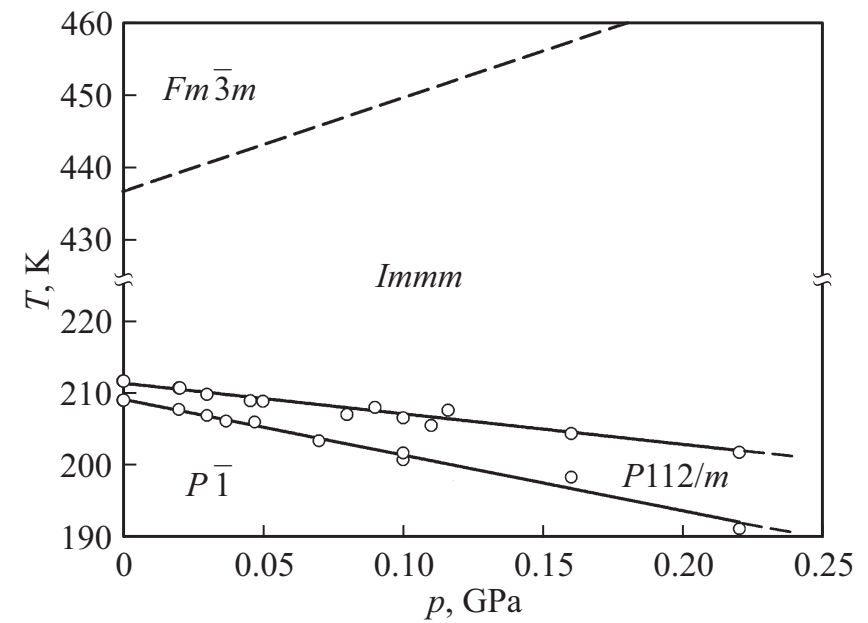

Рис. 4. Фазовая диаграмма температура-давление кристалла $\left(\mathrm{ND}_{4}\right)_{3} \mathrm{VO}_{2} \mathrm{~F}_{4}$. Линия раздела фаз $F m \overline{3} m$ и $\mathrm{Immm}$ (штриховая линия) восстановлена на основании расчета $\left(d T_{1} / d p\right)_{\text {расч. }}$

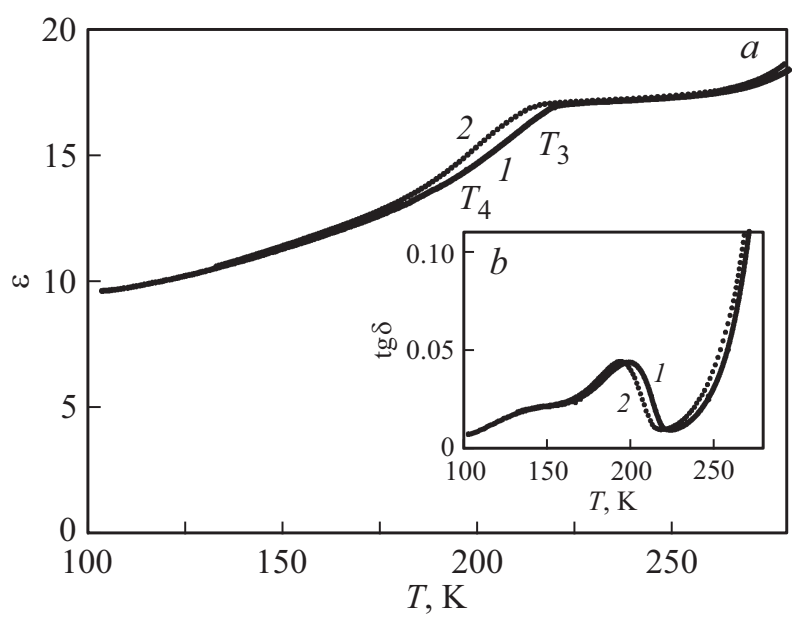

Рис. 5. Температурные зависимости диэлектрической проницаемости $\varepsilon(T)(a)$ и диэлектрических потерь $\operatorname{tg} \delta(T)(b)$, измеренных в режимах нагрева (1) и охлаждения (2).

тур фазовых переходов со значительно отличающимися отрицательными барическими коэффициентами.

Исследования диэлектрических свойств в диапазоне температур $T \approx 100-300 \mathrm{~K}$ проводились с помощью измерителя иммитанса Е7-20 $(v=1 \mathrm{kHz})$ в режимах нагрева и охлаждения $(d T / d t=0.7 \mathrm{~K} / \mathrm{min})$. На образцы для измерений $\varepsilon(T)$, приготовленные в виде спрессованных $(\Delta p \approx 2 \mathrm{GPa})$ без термической обработки таблеток $(d=8 \mathrm{~mm}, h=0.7-1 \mathrm{~mm})$, наносились электроды из сусального золота.

На температурной зависимости диэлектрической проницаемости наблюдается аномальное поведение в области фазовых переходов при $T_{3}$ и $T_{4}$, связанное со следующими небольшими изменениями диэлектрической проницаемости $\Delta \varepsilon_{3}=1.7$ и $\Delta \varepsilon_{4}=1.2$ (рис. 5,a). Температурная зависимость $\operatorname{tg} \delta$ также характеризуется сложным аномальным поведением ниже и выше температур $T_{3}$ и $T_{4}$, свидетельствующим о росте диэлектрических потерь (рис. 5,b). Именно поэтому надежные измерения в области фазового перехода при $T_{1}$ были затруднены. Зависимостям $\varepsilon(T)$ и $\operatorname{tg} \delta(T)$ свойственны значительные гистерезисные явления в области фазовых переходов Immm $\leftrightarrow P 112 / m$ и $P 112 / m \leftrightarrow P \overline{1}$.

\section{3. Обсуждение результатов}

Сравнительный анализ данных, полученных при исследовании $\left(\mathrm{ND}_{4}\right)_{3} \mathrm{VO}_{2} \mathrm{~F}_{4}$ в настоящей работе и для $\left(\mathrm{NH}_{4}\right)_{3} \mathrm{VO}_{2} \mathrm{~F}_{4}$ в [19], позволяет сделать ряд выводов относительно влияния дейтерирования на фазовые переходы, наблюдавшиеся в протонированном соединении, и, в частности, на энтропийные и барические параметры.

Высокая степень дейтерирования ( 87\% D) не повлияла на симметрию кристалла диоксотетрафторида ванадия при комнатной температуре - в $\left(\mathrm{ND}_{4}\right)_{3} \mathrm{VO}_{2} \mathrm{~F}_{4}$ она осталась ромбической (пр.гр. Immm). Однако при этом произошло существенное увеличение объема элементарной ячейки $(\sim 1.5 \%)$ по сравнению с протонированным ванадатом [17]. Следует заметить, что характер и степень связанности анионных полиэдров, по-видимому, играют существенную роль в формировании размерных параметров кристаллической решетки. Замещение $D \rightarrow H$ в оксифторидах с изолированными октаэдрами, $\left(\mathrm{NH}_{4}\right)_{2} \mathrm{WO}_{2} \mathrm{~F}_{4}$ и $\left(\mathrm{NH}_{4}\right)_{2} \mathrm{MoO}_{2} \mathrm{~F}_{4}$, приводило к уменьшению объема элементарной ячейки на $\sim 0.2 \%[25,26]$.

Не обнаружено аномалий в поведении исследованных термодинамических свойств $\left(\mathrm{ND}_{4}\right)_{3} \mathrm{VO}_{2} \mathrm{~F}_{4}$, которые могли бы быть связаны с фазовым переходом Іттm $\leftrightarrow$ ромбическая фаза, наблюдавшимся в $\left(\mathrm{NH}_{4}\right)_{3} \mathrm{VO}_{2} \mathrm{~F}_{4}$ при $T_{2}$ (табл. 2) [18,19]. То есть изменение химического давления, связанное с увеличением объема ячейки, привело к реализации прямого перехода Immm $\leftrightarrow P 112 / m$ в $\left(\mathrm{ND}_{4}\right)_{3} \mathrm{VO}_{2} \mathrm{~F}_{4}$.

Интегрирование температурных зависимостей аномальных теплоемкостей (рис. 2,a и рис. 6,a) позволило установить величины и поведение энтропии, связанной с последовательностью фазовых переходов $\Delta S_{i}(T)=\int\left(\Delta C_{p} / T\right) d T$ (рис. $\left.2, b\right)$. Вследствие удаленности структурного превращения $F m \overline{3} m \leftrightarrow I m m m$ от низкотемпературных переходов (рис. 2, $a$ ) не составляло труда определить изменение энтропии при $T_{1}$ (табл. 2). Для переходов $I m m m \leftrightarrow P 112 / m$ и $P 112 / m \leftrightarrow \bar{P} 1$, реализующихся при очень близких температурах $T_{3}$ и $T_{4}$, на первом этапе было установлено только суммарное изменение энтропии $\Delta S_{3}+\Delta S_{4}=5.8 \pm 0.5 \mathrm{~J} / \mathrm{mol} \cdot \mathrm{K}$. Для определения индивидуальных энтропий мы воспользовались подходом, успешно примененным нами ранее для выделения аномальных теплоемкостей в фазах $P 112 / m$ и $P \overline{1}$ оксифторида $\left(\mathrm{NH}_{4}\right)_{3} \mathrm{VO}_{2} \mathrm{~F}_{4}[19]$.

Наличие избыточной теплоемкости в довольно широких интервалах температур ниже $T_{3}$ и $T_{4}$ (рис. 6, $a$ ) позволяет выполнить анализ ее поведения в рамках 


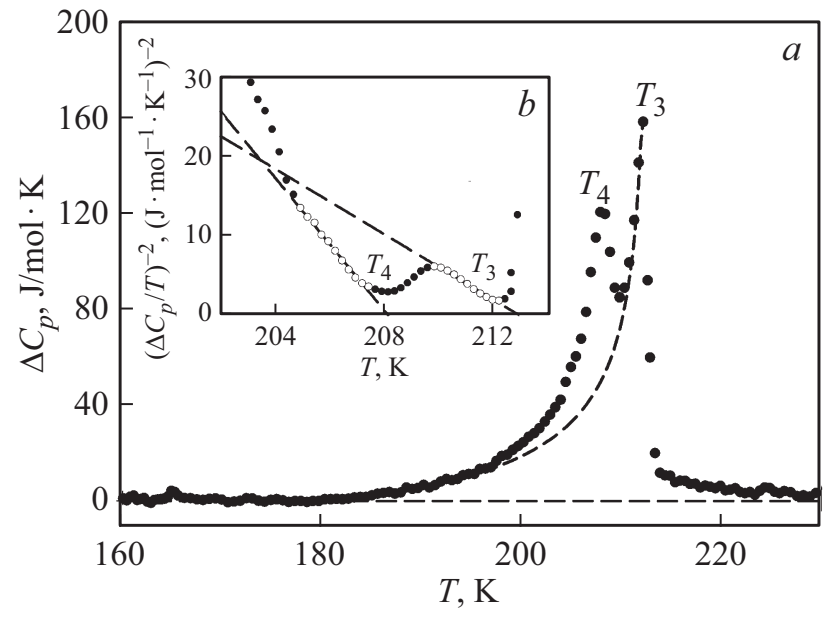

Рис. 6. Температурные зависимости избыточной теплоемкости $(a)$ и квадрата обратной ее величины $(b)$ в окрестности $T_{3}$ и $T_{4}$.

термодинамической теории Ландау. В соответствии с одним из следствий этой теории [28] квадрат обратной избыточной теплоемкости является линейной функцией температуры: $\quad\left(\Delta C_{p} / T\right)^{-2}=\left[2 \cdot\left(B^{2}-3 \cdot A^{\prime} C\right)^{0.5} / A_{T}^{2}\right]^{2}+$ $+12 \cdot C\left(T_{0}-T\right) / A_{T}^{3}$. Здесь величины $A_{T} \cdot\left(T_{0}-T_{C}\right)+$ $+A_{T} \cdot\left(T-T_{0}\right)=A^{\prime}+A_{T} \cdot\left(T-T_{0}\right)=A, B \quad$ и $C$ являются коэффициентами термодинамического потенциала: $\Delta \Phi(p, T, \eta)=A \cdot \eta^{2}+B \cdot \eta^{4}+C \cdot \eta^{6}(\eta-$ параметр перехода, $T_{C}$ - температура Кюри, $T_{0}-$ температура фазового перехода).

Данные, представленные на рис. 6, $b$, показывают, что ниже $T_{3}$ и $T_{4}$ температурное поведение величины квадрата обратной избыточной теплоемкости $\left(\mathrm{ND}_{4}\right)_{3} \mathrm{VO}_{2} \mathrm{~F}_{4}$ действительно описывается линейными зависимостями от температуры. Это позволило выполнить процедуру разделения индивидуальных вкладов аномальных теплоемкостей путем экстраполяции зависимости $\left(\Delta C_{p} / T\right)^{-2}(T)$, связанной с превращением Immm $\leftrightarrow P 112 / m$, на область температур ниже $T_{4}$ (рис. $6, a$ ) и, таким образом, установить индивидуальные энтропии фазовых переходов $\Delta S_{3}$ и $\Delta S_{4}$ (табл. 2).

Из зависимостей $\left(\Delta C_{p} / T\right)^{-2}(T)$ были также определены соотношения между коэффициентами термодинамического потенциала $A_{T}^{2} / B$ и $A_{T}^{3} / C$, которые использовались для оценки размерных $T_{i}-T_{C i}$ и безразмерных $N_{i}= \pm\left[B^{2} /\left(3 \cdot A_{T} \cdot C \cdot T_{C i}\right)\right]^{-0.5}$ параметров, характеризующих степень близости фазовых переходов к трикритической точке (табл. 2). Скачкообразные изменения энтропии (рис. 2, $b$ ) и деформации (рис. 3, $a$ ) при фазовых переходах свидетельствуют о том, что $\left(\mathrm{ND}_{4}\right)_{3} \mathrm{VO}_{2} \mathrm{~F}_{4}$, претерпевает превращения первого рода. В соответствии с этим вычисленные значения $N_{i}$ для переходов Immm $\leftrightarrow P 112 / m$ и $P 112 / m \leftrightarrow P \overline{1}$ имеют отрицательный знак (табл. 2). Из сравнения размерных и безразмерных параметров следует, что переход при $T_{4}$ в $\left(\mathrm{ND}_{4}\right)_{3} \mathrm{VO}_{2} \mathrm{~F}_{4}$ существенно ближе к трикритической точке, которая характеризуется условиями $T_{i}-T_{C i}=0$ и $N=0$. Дейтерирование, с одной стороны, незначительно повлияло на параметры $T_{3}-T_{C 3}$ и $N_{3}$, а с другой стороны, привело к приближению превращения при $T_{4}$ к трикритической точке.

Как было сказано выше, по техническим причинам невозможно было экспериментально исследовать восприимчивость к давлению кристалла $\left(\mathrm{ND}_{4}\right)_{3} \mathrm{VO}_{2} \mathrm{~F}_{4}$ в области высокотемпературного фазового перехода $F m \overline{3} m \leftrightarrow I m m m$. Однако наличие сведений о скачках энтропии $\delta S_{1} \approx 4.8 \mathrm{~J} / \mathrm{mol} \cdot \mathrm{K}$ (рис. $2, b$ ) и деформации $\delta\left(\Delta V / V_{0}\right)_{T 1}=0.006$ (рис. 3, $\left.a\right)$ позволяют рассчитать соответствующий барический коэффициент $\left(d T_{1} / d p\right)_{\text {расч }}$ (табл. 2), используя уравнение Клапейрона-Клаузиуса.

Для низкотемпературных фазовых переходов, имеющих место в кристалле $\left(\mathrm{ND}_{4}\right)_{3} \mathrm{VO}_{2} \mathrm{~F}_{4}$ при $T_{3}$ и $T_{4}$, барические коэффициенты $d T_{i} / d p$, были определены двумя способами: во-первых, прямыми измерениями сдвига

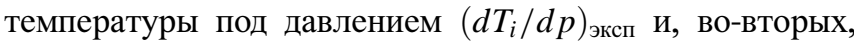
путем расчета $\left(d T_{i} / d p\right)_{\text {расч }}$ в рамках соотношения Пиппарда $C_{p}=\beta \cdot V \cdot T_{i} /\left(d T_{i} / d p\right)_{p=0}+$ const [29] (табл. 2), используя экспериментальные зависимости $C_{p}(T)$ и $\beta(T)$. Принимая во внимание трудности, возникающие при определении корректных температурных зависимостей $\beta$ при температурах ниже $T_{3}$ и $T_{4}$, согласие между экспериментальными и рассчитанными барическими коэффициентами можно считать удовлетворительным, что также свидетельствует о надежности результатов независимых экспериментов.

Барический коэффициент для перехода $F m \overline{3} m \leftrightarrow I m m m$ в $\left(\mathrm{NH}_{4}\right)_{3} \mathrm{VO}_{2} \mathrm{~F}_{4}$ не был определен [19]. Однако в соответствии с соотношением величин $d T_{3} / d p$ и $d T_{4} / d p$ в обоих ванадатах, с большой долей достоверности можно считать, что, как минимум, $d T_{1} / d p>0$ и для $\left(\mathrm{NH}_{4}\right)_{3} \mathrm{VO}_{2} \mathrm{~F}_{4}$. Таким образом, для обоих оксифторидов, $\left(\mathrm{ND}_{4}\right)_{3} \mathrm{VO}_{2} \mathrm{~F}_{4}$ и $\left(\mathrm{NH}_{4}\right)_{3} \mathrm{VO}_{2} \mathrm{~F}_{4}$, характерно значительное расширение под давлением температурной области существования ромбической фазы Immm.

В дейтерированном кристалле произошли существенное увеличение скоростей понижения температур переходов Immm $\leftrightarrow P 112 / m$ и $P 112 / m \leftrightarrow P \overline{1}$ под давлением и рост их различия (табл. 2). Вполне вероятно, что это может быть связано с отсутствием второй ромбической фазы в $\left(\mathrm{ND}_{4}\right)_{3} \mathrm{VO}_{2} \mathrm{~F}_{4}$. В результате стабильность моноклинной и триклинной фаз с ростом давления сохраняется до более низких температур, а давление, приводящее к подавлению фазы $P \overline{1}$, оказывается значительно ниже для $\left(\mathrm{ND}_{4}\right)_{3} \mathrm{VO}_{2} \mathrm{~F}_{4}(\sim 2.7 \mathrm{GPa})$, чем для $\left(\mathrm{NH}_{4}\right)_{3} \mathrm{VO}_{2} \mathrm{~F}_{4}$ $(\sim 8.2 \mathrm{GPa})$.

В соответствии с данными, приведенными в табл. 2, дейтерирование привело к небольшому изменению температур фазовых превращений $F m \overline{3} m \leftrightarrow I m m m$, Immm $\leftrightarrow P 112 / m$ и $P 112 / m \leftrightarrow P \overline{1}$ по сравнению с протонированным кристаллом. Причем характер изменения температур различный: $T_{1}$ уменьшается на $\sim 1 \mathrm{~K}$, а $T_{3}$ и $T_{4}$ увеличиваются соответственно на $\sim 2 \mathrm{~K}$ и 
$\sim 3 \mathrm{~K}$. Такое изменение температур переходов согласуется с положительным барическим коэффициентом перехода $F m \overline{3} m \leftrightarrow I m m m$ (табл. 2) и фазовой диаграммой температура-давление, охватывающей последовательные превращения Immm $\leftrightarrow P 112 / m \leftrightarrow P \overline{1} \quad$ в кристалле $\left(\mathrm{NH}_{4}\right)_{3} \mathrm{VO}_{2} \mathrm{~F}_{4}$ [19]. Действительно, отмеченное выше увеличение объема элементарной ячейки в дейтерированном ванадате можно рассматривать, как связанное с уменьшением химического давления в кристаллической решетке. Рассчитанная абсолютная величина отрицательного давления $(\Delta p \approx-0.08 \mathrm{GPa})$ для кристалла $\left(\mathrm{ND}_{4}\right)_{3} \mathrm{VO}_{2} \mathrm{~F}_{4}$ оказалась существенно больше давления $(\Delta p \approx 0.02 \mathrm{GPa})$, возникающего при дейтерировании аммонийного оксифторида с изолированными октаэдрами $\left(\mathrm{ND}_{4}\right)_{2} \mathrm{MoO}_{2} \mathrm{~F}_{4}[25,26]$. Не исключено, что значительная величина $\Delta p$ может быть причиной выше отмеченного отсутствия аномалии физических свойств $\left(\mathrm{ND}_{4}\right)_{3} \mathrm{VO}_{2} \mathrm{~F}_{4}$, наблюдавшейся в протонированном кристалле при $T_{2} \approx 244 \mathrm{~K}$ и связанной с фазовым превращением Immm $\leftrightarrow$ ромбическая фбаза [19].

Вследствие сложного двойникования кристалла результаты поляризационно-оптических исследований не позволили сделать однозначного заключения о сегнетоэластической природе фазовых переходов в $\left(\mathrm{NH}_{4}\right)_{3} \mathrm{VO}_{2} \mathrm{~F}_{4}$ [18]. Однако выбор центросимметричных пространственных групп позволяет считать, что физическая природа структурных превращений не является сегнетоэлектрической. В пользу этого свидетельствовали также данные диэлектрических исследований: небольшие аномалии на зависимостях $\varepsilon(T)$ [19] в соответствии c [30] интерпретировались как связанные с искажениями структуры, не сопровождающимися возникновением полярных фаз. В настоящей работе не обнаружено значительных аномалий диэлектрической проницаемости и тангенса угла потерь и для $\left(\mathrm{ND}_{4}\right)_{3} \mathrm{VO}_{2} \mathrm{~F}_{4}$. Таким образом, природа фазовых переходов в комплексных фторкислородных ванадатах не зависит от состава тетраэдрического полиэдра.

Дейтерирование привело к некоторому уменьшению индивидуальных энтропий фазовых переходов $F m \overline{3} m \leftrightarrow I m m m$ и $\quad$ Immm $\leftrightarrow P 112 / m$ и в пределах экспериментальной ошибки не вызвало изменений энтропии перехода типа смещения $P 112 / m \leftrightarrow P \overline{1}$ (табл. 2). В результате произошло уменьшение и суммарных изменений энтропии $\Sigma \Delta S_{i}$, связанных с искажением структуры при понижении симметрии кристаллической решетки от кубической $F m \overline{3} m$ до триклинной $P \overline{1}: \Sigma \Delta S_{i} \approx 14.5 \pm 1.7 \mathrm{~J} / \mathrm{mol} \cdot \mathrm{K}=$ $=(1.74 \pm 0.20) \cdot R$ для $\left(\mathrm{ND}_{4}\right)_{3} \mathrm{VO}_{2} \mathrm{~F}_{4}$ и $\Sigma \Delta S_{i} \approx 16.9 \pm$ $\pm 1.9 \mathrm{~J} / \mathrm{mol} \cdot \mathrm{K}=(2.03 \pm 0.23) \cdot R$ для $\left(\mathrm{NH}_{4}\right)_{3} \mathrm{VO}_{2} \mathrm{~F}_{4}[19]$.

Среди аммонийных оксифторидов $\left(\mathrm{NH}_{4}\right)_{3} \mathrm{VO}_{2} \mathrm{~F}_{4}$ является замечательным исключением, так как для него, во-первых, определены сингонии всех фаз [18] и, во-вторых, уточнена структура первой искаженной $\operatorname{Immm}(I 222)$ фазы $[17,20]$. Симметрия кубической фазы $F m \overline{3} m$ для $\left(\mathrm{NH}_{4}\right)_{3} \mathrm{VO}_{2} \mathrm{~F}_{4}$ была выбрана по аналогии с родственным ванадатом $\left(\mathrm{NH}_{4}\right)_{3} \mathrm{VOF}_{5}$, для которого уточнена структура выше $T_{1}$ [20]. При анализе структуры предпочтение было отдано модели, в которой центральный атом $V$ смещен по направлению к ребру октаэдра, образованному двумя атомами кислорода, так как этот вариант разупорядочения приводит к некоторому понижению $R$-фактора. В этом случае все анионные полиэдры должны иметь 12 пространственных ориентаций в фазе $F m \overline{3} m$. Аммонийные группы также считаются разупорядоченными, но с разной степенью беспорядка: в позициях $8 c$ (две третьих тетраэдров) и $4 b$ (одна треть тетраэдров) тетраэдры имеют соответственно четыре и восемь пространственных ориентаций [20].

В рамках этой структурной модели полное упорядочение анионных и катионных полиэдров должно сопровождаться изменением энтропии: $R \cdot \ln 12+2 / 3 R \cdot \ln 4$ $+1 / 3 R \cdot \ln 8=34.1 \mathrm{~J} / \mathrm{mol} \cdot \mathrm{K}=R \cdot \ln 60$, которое значительно превышает экспериментально установленные значения: $\Sigma \Delta S_{i} \approx R \cdot \ln 6$ для $\left(\mathrm{ND}_{4}\right)_{3} \mathrm{VO}_{2} \mathrm{~F}_{4} \quad$ и $\Sigma \Delta S_{i} \approx R \cdot \ln 8$ для $\left(\mathrm{NH}_{4}\right)_{3} \mathrm{VO}_{2} \mathrm{~F}_{4}$.

Можно было бы предполагать, что это различие обусловлено существованием дополнительных фазовых переходов при более низких температурах, по сравнению с достигнутыми в калориметрических экспериментах $(\sim 100 \mathrm{~K})$. Действительно, несмотря на триклинную симметрию фазы ниже $T_{4}$, вполне можно было допустить существование изменения структуры с повышением симметрии при понижении температуры, как это, например, наблюдалось в двойных солях фторидов с аналогичной исходной фазой $F m \overline{3} m$ [31]. Однако при исследованиях спектров КР и ИК вплоть до $T \approx 8 \mathrm{~K}$ не обнаружено каких-либо доказательств в пользу существования фазовых переходов помимо известных [32].

Следует заметить, что изменения энтропии в исследованных ванадатах удовлетворительно согласуются с аналогичными параметрами родственных аммонийных соединений с исходной кубической симметрией $F m \overline{3} m$, но с другим составом и симметрией фтор-кислородных октаэдров $C_{4 v}-\left(\mathrm{NH}_{4}\right)_{3} \mathrm{TiOF}_{5}\left(\Sigma \Delta S_{i} \approx 2.18 \cdot R\right)$; $C_{3 v}-\left(\mathrm{NH}_{4}\right)_{3} \mathrm{WO}_{3} \mathrm{~F}_{3} \quad\left(\Sigma \Delta S_{i} \approx 2.03 \cdot R\right) \quad[33]$, для которых уточнение структуры искаженных фаз не выполнено из-за возникновения сложной двойниковой структуры при фазовых переходах [34]. Сравнение этих данных с кубическими фторидами, претерпевающими значительные искажения структуры в результате последовательных: $F m \overline{3} m \leftrightarrow P 2_{1} / n \leftrightarrow I 2 / m \leftrightarrow I \overline{1}$ $\left(\left(\mathrm{NH}_{4}\right)_{3} \mathrm{ScF}_{6}-\Sigma \Delta S_{i}=23 \mathrm{~J} / \mathrm{mol} \cdot \mathrm{K}=2.77 \cdot R\right)$ или прямых $F m \overline{3} m \leftrightarrow I \overline{1} \quad\left(\left(\mathrm{NH}_{4}\right)_{3} \mathrm{GaF}_{6}-\Sigma \Delta S=23 \mathrm{~J} / \mathrm{mol} \cdot \mathrm{K}\right.$ $=2.77 \cdot R)$ фазовых переходов [35], свидетельствует о том, что степень разупорядочения структурных элементов в комплексных фторидах существенно больше. Таким образом, на этот параметр оказывает влияние симметрия шестикоординированного анионного полиэдра, которая является более высокой во фторидах $\left(O_{h}\right)$, чем в ванадиевых оксифторидах $\left(C_{2 v}\right)$. Действительно, результаты многочисленных экспериментальных калориметрических исследований показали, что максимальные величины энтропии фазовых переходов в аммоний- 
ных оксифторидах и фторидах, каркас кристаллической решетки которых образован шестикоординированными анионными полиэдрами, не превышают соответственно $\Sigma \Delta S_{i}=2.1 \mathrm{R}$ и $\Sigma \Delta S_{i}=2.8 \mathrm{R}[23]$.

$\mathrm{C}$ нашей точки зрения, гигантские изменения энтропии, следующие из модельных представлений о разупорядочении всех структурных элементов в фазе $F m \overline{3} m$, следует рассматривать, как предельно возможные. И это обстоятельство представляет несомненный интерес с позиций возможности создания высокоэффективных твердотельных барокалорических хладагентов, для которых очень важным параметром является большая величина энтропии фазового перехода [36], т. е. нужно искать пути воздействия на степень разупорядочения структурных элементов. Значительные различия экспериментальных и модельных энтропий фазовых переходов в комплексных оксифторидах позволяют предположить, что для некоторых критических ионов характерен скорее значительный ангармонизм колебаний, чем разупорядочение по нескольким кристаллографическим положениям. В этом случае изменение энтропии пропорционально параметру ангармоничности $\delta=\langle x\rangle^{2} / a^{2}$, который связывает амплитуду колебаний иона $x$ со средним межатомным расстоянием $a$ [37], и может оказаться существенно меньше величины, следующей из модели порядок-беспорядок. В связи с этим следует обратить внимание на то, что уменьшение энтропий в $\left(\mathrm{ND}_{4}\right)_{3} \mathrm{VO}_{2} \mathrm{~F}_{4}$ по сравнению с $\left(\mathrm{NH}_{4}\right)_{3} \mathrm{VO}_{2} \mathrm{~F}_{4}$ (табл. 2) произошло только для фазовых переходов типа порядок-беспорядок при $T_{1}$ и $T_{3}$. Энтропия $\Delta S_{4}$ для перехода типа смещения практически не изменилась. Таким образом, не исключено, что дейтерирование привело к уменьшению степени ангармонизма колебаний тетраэдрических катионов.

\section{4. Заключение}

Выращены кристаллы дейтерированного диоксотетрафторида ванадия $(V),\left(\mathrm{ND}_{4}\right)_{3} \mathrm{VO}_{2} \mathrm{~F}_{4}$ и выполнены исследования теплофизических свойств в широком интервале температур.

Установлено значительное увеличение объема элементарной ячейки $\left(\mathrm{ND}_{4}\right)_{3} \mathrm{VO}_{2} \mathrm{~F}_{4}$ по сравнению с $\left(\mathrm{NH}_{4}\right)_{3} \mathrm{VO}_{2} \mathrm{~F}_{4}(\sim 1.5 \%)$, что привело к изменению химического давления, вызвавшему ряд изменений в картине структурных превращений, имевших место в протонированном ванадате.

1) Вторая ромбическая фаза в $\left(\mathrm{ND}_{4}\right)_{3} \mathrm{VO}_{2} \mathrm{~F}_{4}$ выклинивается и вместо последовательных фазовых переходов Iттm $\leftrightarrow$ ромбическая фаза $\leftrightarrow P 112 / m$ имеет место прямой переход Immm $\leftrightarrow P 112 / m$.

2) Сравнение размерных $T_{0}-T_{C}$ и безразмерных $N$ параметров свидетельствует о том, что переход при $T_{4}$ существенно ближе к трикритической точке и в значительной мере приближается к ней в результате дейтерирования.
3) Определены барические коэффициенты и построена фазовая диаграмма температура-давление, из которой следует, что рост давления сопровождается расширением температурной области устойчивости ромбической фазы Immm и более быстрым подавлением триклинной фазы в $\left(\mathrm{ND}_{4}\right)_{3} \mathrm{VO}_{2} \mathrm{~F}_{4}$. Характер изменения температур фазовых переходов в результате замещения $D \rightarrow H$ согласуется с уменьшением химического давления, обусловленного увеличением объема элементарной ячейки.

4) Диэлектрические исследования показали, что, несмотря на все изменения, физических свойств в результате дейтерирования, природа фазовых переходов осталась несегнетоэлектрической.

5) Суммарные экспериментальные энтропии переходов при $T_{1}$ и $T_{3}$ в $\left(\mathrm{ND}_{4}\right)_{3} \mathrm{VO}_{2} \mathrm{~F}_{4}\left(\Sigma \Delta S_{i} \approx R \cdot \ln 6\right)$ и в $\left(\mathrm{NH}_{4}\right)_{3} \mathrm{VO}_{2} \mathrm{~F}_{4}\left(\Sigma \Delta S_{i} \approx R \cdot \ln 8\right)$ оказались значительно меньше энтропий, следующих из модели разупорядочения структурных элементов в фазе $F m \overline{3} m$ $\left(\Sigma \Delta S_{i} \approx R \cdot \ln 60\right)$ [20], которые, скорее всего, можно рассматривать как предельно возможные для фторкислородных ванадатов. Уменьшение полной энтропии $\Sigma \Delta S_{i}$ в результате дейтерирования с большой долей вероятности свидетельствует в пользу значительного ангармонизма (но не предельного, соответствующего разупорядочению) колебаний аммонийных тетраэдров.

\section{Список литературы}

[1] H.-Y. Chang, S.-H. Kim, P.S. Halasyamani, K.M. Ok. J. Am. Chem. Soc. 131, 2426 (2009).

[2] E.C. Glor, S.M. Blau, J. Yeon, M. Zeller, P. Shiv Halasyamani, J. Schrier, A.J. Norquist. J. Solid State Chem. 184, 1445 (2011).

[3] Y. Inaguma, M. Yoshida, T. Katsumata. J. Am. Chem. Soc. 130, 6704 (2008).

[6] P.A. Maggard, A.L. Kopf, C.L. Stern, K.R. Poeppelmeier. Cryst. Eng. Commun. 6, 451 (2004).

[7] P. Halasyamani, K.R. Heier, M.J. Willis, C.L. Stern, K.R. Poeppelmeier, Z. Anorg. Allg. Chem. 622, 479 (1996).

[8] S. Halasyamani, K.R. Heier, C.L. Stern, K.R. Poeppelmeier. Acta Crystallogr. C 53, 1240 (1997).

[9] P.A. Maggard, A.L. Kopf, C.L. Stern, K.R. Poeppelmeier, K.M. Ok, P.S. Halasyamani. Inorg. Chem. 41, 4852 (2002).

[10] R. Gautier, M.D. Donakowski, K.R. Poeppelmeier. J. Solid State Chem. 195, 132 (2012).

[11] D.W. Aldous, P. Lightfoot. Solid State Sci. 11, 315 (2009).

[12] R.A.F. Pinlac, C.L. Stern, K.R. Poeppelmeier. Crystals 1, 3 (2011).

[13] R. Gautier, M.D. Donakowski, K.R. Poeppelmeier. J. Solid State Chem. 195, 132 (2012).

[14] N.F. Stephens, M. Buck, P.J. Lightfoot. J. Mater. Chem., 15, 4298 (2005).

[15] R.R. Ryan, S.H. Mastin, M.J. Reisfeld. Acta Crystallogr. B 27, 1270 (1971).

[16] S.J. Patwe, A.S. Nagabhusan, K.G. Girija, C.G. Sivan Pillai, A.K. Tyagi. J. Mater. Res. 25, 1251 (2010).

[17] M. Leimkühler, R.J. Mattes. Solid State Chem. 65, 260 (1986). 
[18] С.В. Мельникова, А.Г. Кочарова. ФТТ 51, 3, 562 (2009).

[19] V.D. Fokina, M.V. Gorev, A.G. Kocharova, E.I. Pogoreltsev, I.N. Flerov. Solid State Sci. 11, 836 (2009).

[20] A.A. Udovenko, E.I. Pogoreltsev, Y.V. Marchenko, N.M. Laptash. Acta Cryst. B 73, 1 (2017).

[21] R.L. Withers, F.J. Brink, Yu. Liu, L. Nore'n. Polyhedron 26, 290 (2007).

[22] A.A. Udovenko, N.M. Laptash. Acta Cryst. B 67, 447 (2011).

[23] I.N. Flerov, M.V. Gorev, M.S. Molokeev, N.M. Laptash. Photonic and Electronic Properties of Fluoride Materials (Progress in Fluorine Science Series) / Ed. A. Tressaud, K. Poeppelmeier. Elsevier, Amsterdam (2016). P. 355-81.

[24] Е.В. Богданов, А.Д. Васильев, И.Н. Флеров, Н.М. Лапташ. ФТТ 53, 2, 284 (2011).

[25] И.Н. Флёров, В.Д. Фокина, М.В. Горев, Е.В. Богданов, М.С. Молокеев, А.Ф. Бовина, А.Г. Кочарова. ФТТ 49, 6, 1093 (2007).

[26] E.V. Bogdanov, E.I. Pogoreltsev, M.V. Gorev, I.N. Flerov. Inorg. Chem. 56, 11, 6706 (2017).

[27] Bruker AXS TOPAS V4: General profile and structure analysis software for powder diffraction data. - User's Manual. Bruker AXS, Karlsruhe, Germany. (2008).

[28] К.С. Александров, И.Н. Флёров. ФТТ 21, 327 (1979).

[29] Н. Парсонидж, Л. Стейвли. Беспорядок в кристаллах. Мир, М. (1982). 436 с.

[30] Б.А. Струков, А.П. Леванюк. Физические основы сегнетоэлектрических явлений. Наука, М. (1983). 83 с.

[31] M. Molokeev, S.V. Misjul, I.N. Flerov, N.M. Laptash. Acta Cryst. B 70, 924 (2014).

[32] Yu.V. Gerasimova, A.S. Oreshonkov, N.M. Laptash, A.N. Vtyurin, A.S. Krylov, N.P. Shestakov, A.A. Ershov, A.G. Kocharova. Spectrochim. Acta A 176, 106 (2017).

[33] И.Н. Флёров, М.В. Горев, В.Д. Фокина, А.Ф. Бовина, H.M. Лапташ. ФТТ 46, 888 (2004).

[34] A.A. Udovenko, N.M. Laptash. Acta Cryst. B 67, 447 (2011).

[35] M.V. Gorev, I.N. Flerov, A. Tressaud. J. Phys.: Condens. Matter 11, 7493 (1999).

[36] M.V. Gorev, E.V. Bogdanov, I.N. Flerov. J. Phys D 50, 384002 (2017).

[37] В.Г. Вакс. Введение в микроскопическую теорию сегнетоэлектриков. Наука, М. (1973). 327 с.

Редактор Т.Н. Василевская 